\title{
Nature-Inspired Algorithms and Applications: Selected Papers from CIS2013
}

\author{
Yuping Wang, ${ }^{1}$ Yiu-ming Cheung, ${ }^{2}$ Xiao-Zhi Gao, ${ }^{3}$ Patrick Wang, ${ }^{4}$ and Hailin Liu ${ }^{5}$ \\ ${ }^{1}$ School of Computer Science and Technology, Xidian University, Xian 710071, China \\ ${ }^{2}$ Department of Computer Science, Hong Kong Baptist University, Hong Kong \\ ${ }^{3}$ Department of Electrical Engineering and Automation, Aalto University School of Electrical Engineering, 00076 Espoo, Finland \\ ${ }^{4}$ College of Computer Science, Northeastern University, Boston, MA 02115, USA \\ ${ }^{5}$ School of Applied Mathematics, Guangdong University of Technology, Guangzhou 510520, China
}

Correspondence should be addressed to Yuping Wang; ywang@xidian.edu.cn

Received 26 March 2015; Accepted 26 March 2015

Copyright (c) 2015 Yuping Wang et al. This is an open access article distributed under the Creative Commons Attribution License, which permits unrestricted use, distribution, and reproduction in any medium, provided the original work is properly cited.

With the fast development of computer science and technology, the computational intelligence (CI) methods have been successfully applied to deal with security and trusted problems during the past decades. It has been a necessary and emerging task to cross-fertilize and integrate the ideas and techniques of these two important fields: CI and information security (IS). This special issue selects 11 high-quality extended papers from a total of 171 accepted papers of the 2013 International Conference on Computational Intelligence and Security (CIS2013). These papers can be classified into two groups. The first group contains seven papers developing new CI models with applications in solving real-world problems, and the second group has four papers focusing on the theoretical study of novel CI algorithms.

In the first group, the paper entitled "Emergency Vehicle Scheduling Problem with Time Utility in Disasters" by X. Gan et al. proposes a fuzzy optimization model for the flexible emergency rescue system and uses the particle swarm optimization (PSO) method to handle this model. The paper entitled "Effective Task Scheduling and IP Mapping Algorithm for Heterogeneous NoC-Based MPSoC" by P. Yang and Q. Wang develops a new task scheduling scheme for the NoC(network-on-chip-) based MPSoC (multiprocessor systemon-chip) design process and uses the PSO to acquire the minimum task execution time with the least task running and transfer cost. The paper entitled "Optimal Vaccine Distribution Strategy for Different Age Groups of Population:
A Differential Evolution Algorithm Approach" by X.-M. Hu et al. applies the evolutionary algorithms to find the optimal strategy for the vaccine distribution of different age groups of population. This approach can minimize the number of the infectious individuals during the epidemic outbreak. In one of the papers, W. Wang et al. present an optimization model using a convex risk measure for the portfolio selection problems and design a genetic algorithm on the basis of this effective model. X. Deng and J. Deng establish a machine learning approach for the prisoners' dilemma problem in the game theory and provide an effective method for the incomplete information game problems in their paper. C. Ruan and J. Yang investigate a fuzzy software quality evaluation method via the index system models and fuzzy methods that are applicable to those cases, in which the experts have inconsistent and uncertain judgments for software quality. J. Qu et al. present a new cascade pedestrian detection technique using the orthogonal scene motion pattern model in a general density video and a specifically designed feature classifier. The static classification and surveillance video experiments demonstrate the effectiveness of their method.

In the second group, D. Jiang and Z. Fan suggest a new idea to design algorithms: automatically designing evolutionary algorithms by computers according to the problem requirements. $\mathrm{H}$. Li and L. Fang propose an efficient evolutionary algorithm for handling interval linear bilevel programming problems. $\mathrm{H}$. Li and L. Zhang investigate 
a differential evolution method for the constrained bilevel programming problems by using the hybrid mutations and an improved constraint handling technique, which has been validated in the simulations of some linear and nonlinear bilevel programming problems. R. Wang et al. propose an improved adaptive growing and pruning algorithm (IAGP) for neural networks, and simulation results show that it can quickly and accurately predict the traffic capacity.

By publishing the above interesting papers in this special issue, we wish to provide our readers with some inspiring ideas for developing new CI models with applications in attacking practical problems.

Yuping Wang Yiu-ming Cheung

Xiao-Zhi Gao

Patrick Wang

Hailin Liu 


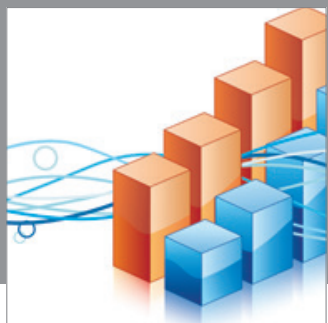

Advances in

Operations Research

mansans

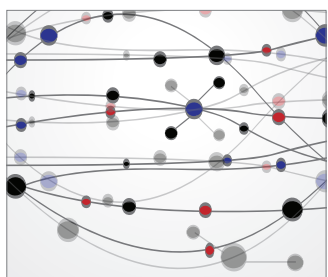

The Scientific World Journal
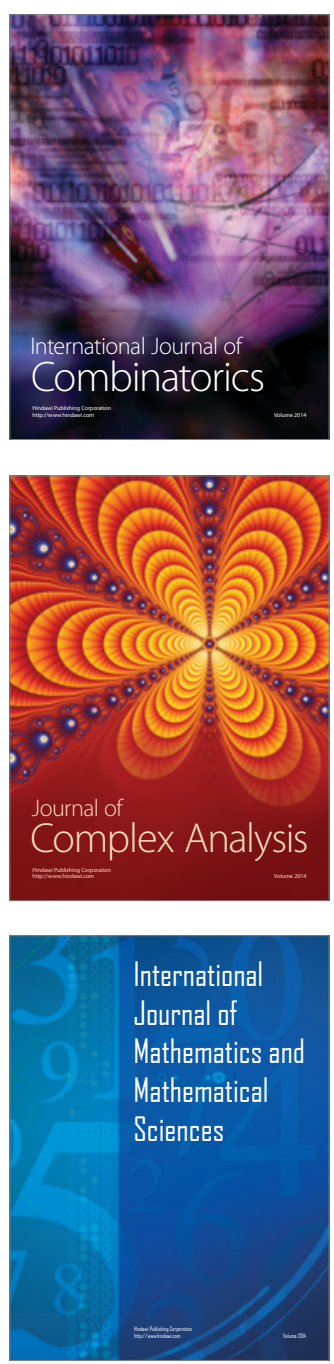
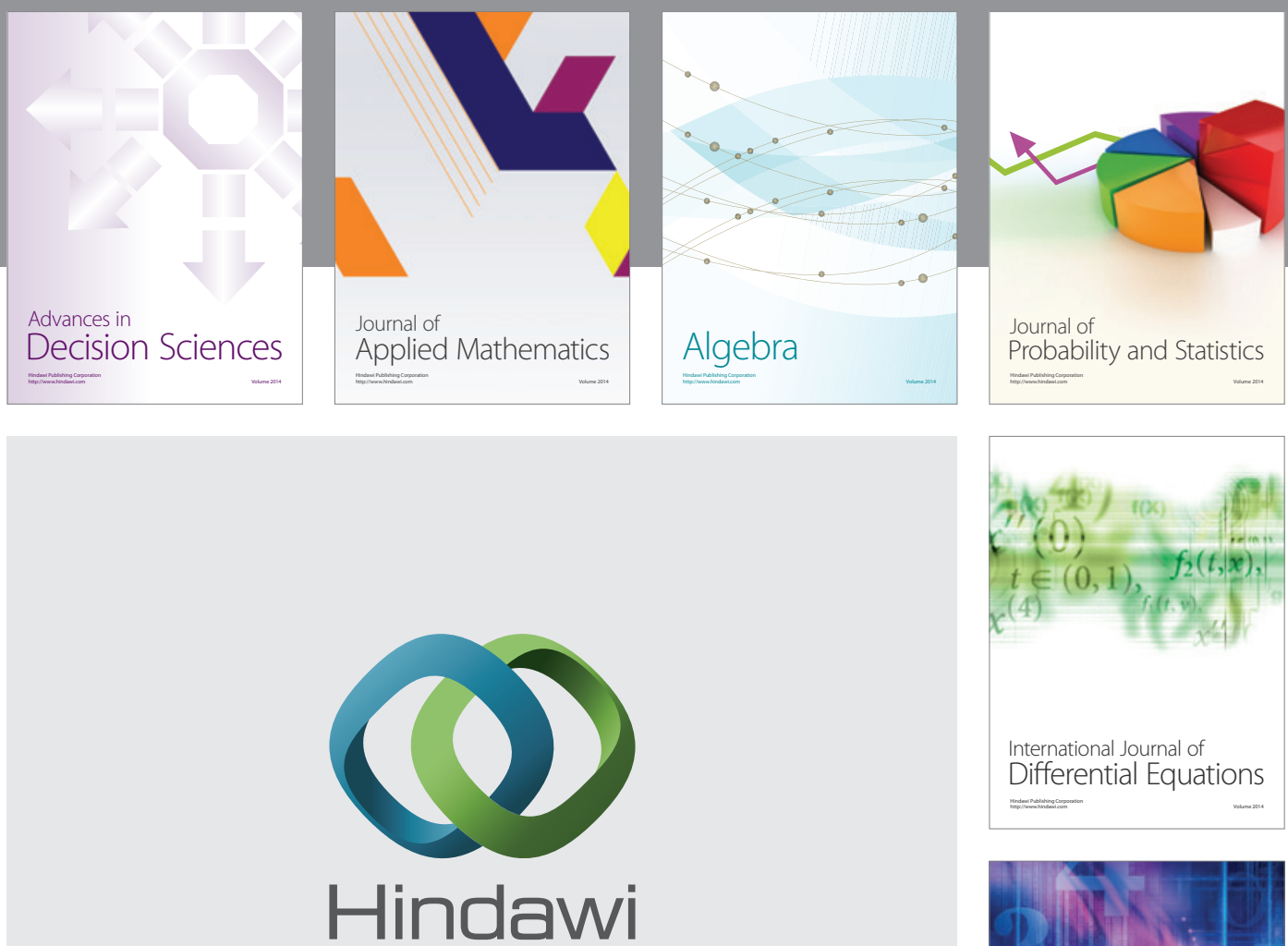

Submit your manuscripts at http://www.hindawi.com
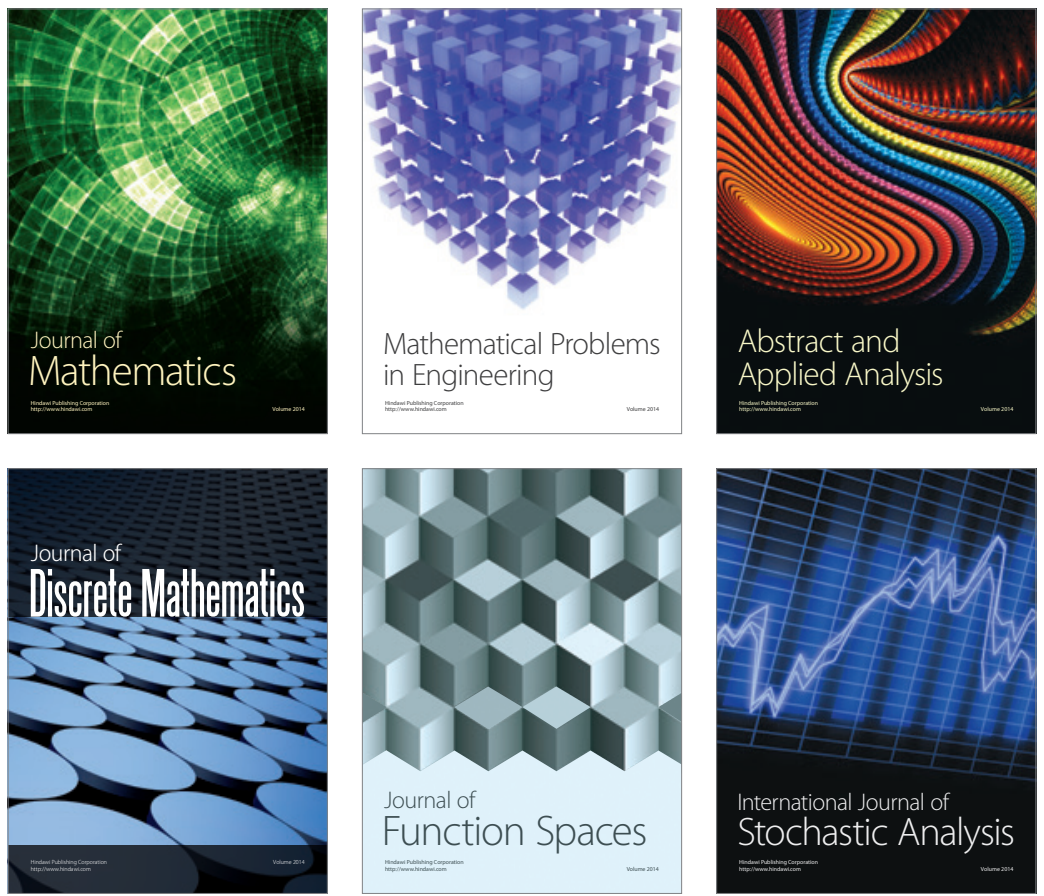

Journal of

Function Spaces

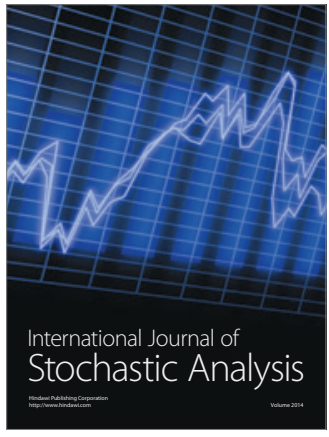

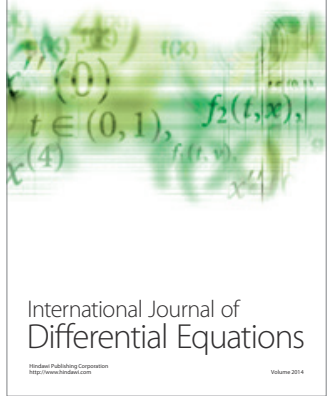
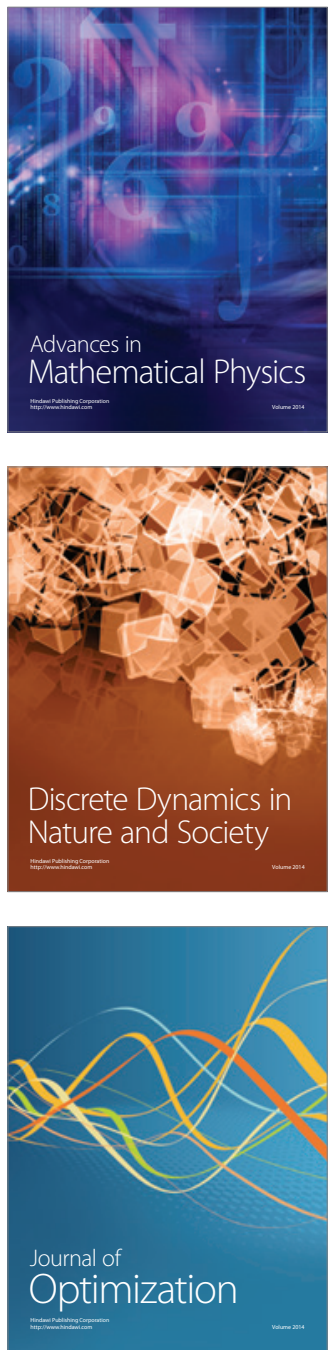